MATEC Web of Conferences 22,01033 (2015)

DOI: $10.1051 /$ matec conf/ 20152201033

(C) Owned by the authors, published by EDP Sciences, 2015

\title{
Performance Analysis of Precoding Based on Massive MIMO System
}

\author{
Yi Li, Junxuan Wang* \& Zhenzhen Gao \\ School of Communication and Information Engineering, Xi'an University of Posts and Telecommunications, \\ Xi'an, Shaanxi, China
}

\begin{abstract}
In order to improve the system performance, the authors consider a single-cell multiuser Massive MIMO downlink time-division duplex (TDD) system for the imperfect channel state information (CSI). For the zero-forcing (ZF) and the matched filtering (MF) precoding scheme, the authors propose a normalization algorithm: the vector normalization. Assume that the channel estimation is used to acquire CSI by using the uplink pilot sequence, and utilize the proposed algorithm to normalize the precoding matrix in the downlink; we derive the achievable sum rate of ZF and MF. Through the analysis and comparison of two precoding schemes' performance, the authors conclude that ZF is better than MF with vector normalization algorithm in the high SNR region; and MF is better than ZF in the low SNR region. Simulation results confirm the above conclusion.
\end{abstract}

Keywords: Massive MIMO; imperfect CSI; precoding; vector normalization; achievable sum rate

\section{INTRODUCTION}

The massive MIMO (multiple-input multiple-output) technology is also called the Large-Scale Antenna Systems or the Large-Scale MIMO. This technology is a large number of base station (BS) transmit antennas, and the user terminal uses a small number of receive antennas to communication. The huge interference of the suppression gain and the array gain caused by the large-scale antenna array which makes the edge user spectral efficiency and the cell total spectral efficiency have been greatly improved ${ }^{[1]}$. Compared with the $4 \mathrm{G}$ wireless technologies, the Massive MIMO brought great energy efficiency and emission spectral efficiency gain. So the massive MIMO technology is a revolutionary technology of the $5 \mathrm{G}$ communication in the future ${ }^{[2-3]}$. In this paper, we recommend a TDD model for the massive MIMO system. In order to improve the performance of MIMO for the frequency division duplex (FDD) model, which required obtaining the CSI of each user, the high consumption of downlink channel of overhead and feedback will ultimately limit the number of the BS antennas ${ }^{[4]}$. However, the TDD system has the channel reciprocity. According to the uplink channel estimation, the BS downlink transmission can effectively reduce the related signaling overhead. Thus, the massive MU-MIMO system using TDD method is a more effective solution ${ }^{[5]}$.

For the precoding algorithm, a lot of literature has been given on the system analysis and theoretical study. [6] proposed beamforming training programs to estimate CSI of each user, and the BS transmits the precoded pilot sequence to all users, then, each user according to the received pilot with minimum mean square error (MMSE) to estimate the effective channel gain. [7] studied the performance of conjugate beam- forming $(\mathrm{CBF})$ and $\mathrm{ZF}$ precoding, which pointed out that the performance of $\mathrm{ZF}$ is better than the $\mathrm{CBF}$ precoding for high spectral efficiency and low energy efficiency; the opposite also holds in low spectral efficiency and high energy efficiency. When the BS antenna serves more users, the calculation of CBF may be larger than ZF. To further maximize the system capacity, [8] presented a network of MIMO algorithm with multiple receiving antennas, which assumes that this network supports up to three users by a relatively small number of transmit antennas. The massive MIMO system has been studied in [9] for a multi-cell scenario, there are some important problems in the multi-cell massive MIMO system, such as the pilot pollution, which must be solved before practical application. The author pointed out the influence of pilot pollution on theory and simulation, and proposed a precoding algorithm of the MMSE estimation to reduce interferences of intra-cell and inter-cell. When the transmitter is equipped with enough antennas, the problem of pilot pollution will be eventually eliminated. However, this assumption is not feasible in practice. In [10], to solve this problem, the authors concluded that the antenna of the proposed framework is 10 times less than the previously mentioned system[9], and obtains the same spectral efficiency. [11] analyzed the performance of different precoding and derived the achievable sum-rate bound of system downlink from the perfect CSI scenario. In this paper, we apply the vector normalization algorithm with the classical ZF and MF precoding for imperfect CSI scenario.

This paper is organized as follows: Section 2 introduces the system model; section 3 derives the sum rate lower bound of precoding schemes; the decision threshold of two precoding is studied in section 4; numerical results are shown in section 5; and conclu-

*Corresponding author: 502709443@qqq.com

This is an Open Access article distributed under the terms of the Creative Commons Attribution License 4.0, which permits unrestricted use, distribution, and reproduction in any medium, provided the original work is properly cited. 
sions are presented in section 6 .

\section{SYSTEM MODEL}

\subsection{Uplink training}

We consider a single-cell massive MIMO system with the TDD model, where the base station equipped with $M$ transmit antennas serves $K$ single-antenna users that share the same time-frequency resource. It is shown in Figure 1. Here, the transmission between base station $M$ antennas and $\mathrm{K}$ users is $M \times K$ Rayleigh fading channel matrix $\mathrm{H}$ with i.i.d.CN $(0,1)$ elements. According to the received orthogonal pilot sequence (which is included in symbols) transmitted by the user terminal, the BS estimates the corresponding channel and designs the downlink precoding matrix by using the obtained channel with the channel reciprocity. Then, the received signal at the BS is expressed as follows:

$Y_{p}=\sqrt{p_{p}} H \Phi^{T}+N$

Where $p_{p}=\tau p_{u}, p_{u}$ is the normalized uplink power The $M \times K$ matrix of uplink user training sequence is denoted by $\Phi$, and $\Phi^{H} \Phi=I_{K}$. And let $\mathrm{N}$ be the additive white Gaussian noise matrix with i.i.d.CN $(0,1)$ entries. In this paper, we ignore the large scale fading for simplicity. The corresponding estimation of $\mathrm{H}$ with MMSE channel estimation is given as follows:

$\hat{H}=\frac{\sqrt{p_{p}}}{1+p_{p}} Y_{p}$

The channel matrix $\mathrm{H}$ can be decomposed as ${ }^{[6]}$ :

$H=\hat{H}+\varepsilon$
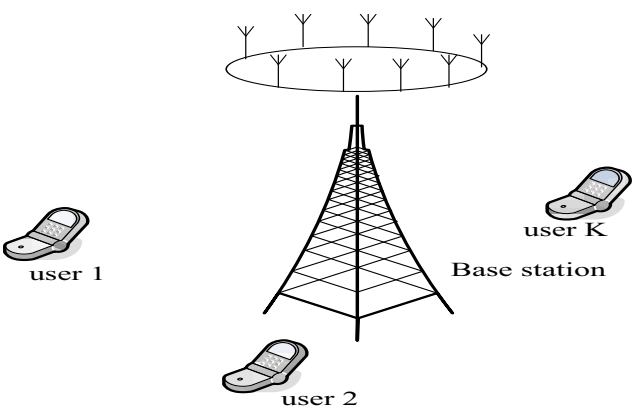

Figure 1. Massive MU-MIMO downlink system model

Where $\varepsilon$ is the channel estimation error. Due to the use of MMSE estimation, $\hat{H}$ and $\varepsilon$ are independent.
Furthermore, $\hat{H}$ has i.i.d.CN $\left(0, \frac{p_{p}}{1+p_{p}}\right)$ elements, and $\varepsilon$ has i.i.d. $\mathrm{CN}_{\left(0, \frac{1}{1+p_{p}}\right)}$.

\subsection{Downlink transmission}

There are two linear precoding techniques usually used in downlink, ZF and MF respectively. The corresponding precoding matrix is expressed as follows:

$$
\begin{aligned}
& F_{Z F}=\hat{H}^{*}\left(\hat{H}^{T} \hat{H}^{*}\right)^{-1}=\left[f_{1}, f_{2}, \ldots, f_{K}\right] \\
& F_{M F}=\hat{H}^{*}=\left[f_{1}, f_{2}, \ldots, f_{K}\right]
\end{aligned}
$$

Where $\mathrm{F}$ is the precoding matrix, and $f_{k}$ is the kth column vector.

In order to satisfy the power control, we need to normalize the precoding matrix. The algorithm as previously mentioned is the vector normalization, and let A be the $M$ based on the $K$ normalization precoding matrix:

$A=\left[\frac{f_{1}}{\left\|f_{1}\right\| \sqrt{K}}, \frac{f_{2}}{\left\|f_{2}\right\| \sqrt{K}}, \ldots, \frac{f_{K}}{\left\|f_{K}\right\| \sqrt{K}}\right]$

Then, in the downlink, the received signal at the user is expressed as follows:

$y=\sqrt{p_{f}} H^{T} A x+n$

Where $\boldsymbol{x}$ is the $K \times 1$ transmit signal vector, and $\boldsymbol{n}$ is the $K \times 1$ vector noise. $p_{f}$ is normalized downlink power that is proportional to the BS radiated power divided by the noise's variance. From (3), we have $h_{k}=\hat{h}_{k}+{ }_{T} \varepsilon_{k}, k=1, \ldots, K$, and let $g_{k i}=\hat{h}_{k}{ }^{T} a_{i}$, $\varphi_{k i}=\varepsilon_{k}{ }^{T} a_{i}$. Then, the received signal at the kth user can be written as follows:

$y_{k}=\sqrt{p_{f}} h_{k}{ }^{T} a_{k} x_{k}+\sqrt{p_{f}} \sum_{i \neq k}^{K} h_{k}{ }^{T} a_{i} x_{i}+n_{k}$

$=\sqrt{p_{f}} \hat{h}_{k}^{T} a_{k} x_{k}+\sqrt{p_{f}} \sum_{i \neq k}^{K} \hat{h}_{k}^{T} a_{i} x_{i}$

$+\sqrt{p_{f}} \sum_{i=1}^{K} \varepsilon_{k}^{T} a_{i} x_{i}+n_{k}$

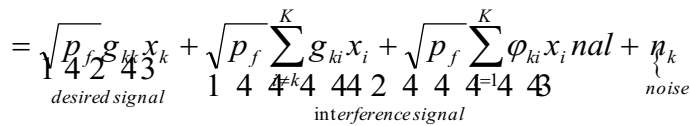

Where $\boldsymbol{a}_{k}, \hat{h}_{k}$ and $\varepsilon_{k}$ are respectively denoted by the $K \times 1$ column vector of $A$, $\hat{H}$ and $\varepsilon$ matrix. 
Form (7), we can obtain the downlink achievable sum rate of kth user which is shown as follows:

$$
R_{\mathrm{k}}=\log _{2}\left(1+\frac{p_{f}\left|g_{k k}\right|^{2}}{1+p_{f} \sum_{i=1, i \neq k}^{K}\left|g_{k i}\right|^{2}+p_{f} \sum_{i=1}^{K}\left|\varphi_{k i}\right|^{2}}\right)
$$

Then, the downlink achievable sum rate of $K$ user can be expressed as follows:

$$
R=\sum_{k=1}^{K} R_{k}
$$

In the following, we use upper and lower bold letters to respectively describe matrices $\mathbf{A}$ and vectors $\boldsymbol{a}$. The superscripts $T, *$ and -1 respectively stand for the transpose, conjugate, and inverse, and $\operatorname{tr}(\mathrm{A})$ is the trace of matrix A. The expectation (variance) operator and the Euclidean norm are respectively denoted by $\mathrm{E}\{\cdot\}(\operatorname{var}\{\cdot\})$ and $\|\cdot\|$.

\section{SUM RATE LOWER BOUND}

\subsection{MF precoding}

Form (9), we can derive the achievable sum rate lower bound of vector normalization with MF precoding which is shown as follows:

$$
\begin{aligned}
& R_{M F}=K \log _{2}\left(1+\frac{p_{f}\left|g_{k k}\right|^{2}}{1+p_{f} \sum_{i=1, i \neq k}^{K}\left|g_{k i}\right|^{2}+p_{f} \sum_{i=1}^{K}\left|\varphi_{k i}\right|^{2}}\right) \\
& \approx K \log _{2}\left(1+\frac{p_{f} \frac{M}{K} \frac{p_{p}}{1+p_{p}}}{1+\frac{K-1}{K} \frac{p_{f} p_{p}}{1+p_{p}}+\frac{p_{f}}{1+p_{p}}}\right) \\
& =K \log \left(1+\frac{M}{K} \frac{p_{f} p_{p}}{\left(1+p_{p}\right)\left(1+p_{f}\right)}\right)
\end{aligned}
$$

Where apply the properties of random vector ${ }^{[12]}$ and the law of lager number, $g_{k i}=\frac{\hat{h}_{k}{ }^{T} \hat{h}_{i}^{*}}{\sqrt{K}\left\|\hat{h}_{i}\right\|}, \varphi_{k i}=\frac{\varepsilon_{k}{ }^{T} \hat{h}_{i}^{*}}{\sqrt{K}\left\|\hat{h}_{i}\right\|}$,

$$
\left|\hat{h}_{k}{ }^{T} \hat{h}_{i}\right|^{2}=\frac{p_{p}{ }^{2}}{\left(1+p_{p}\right)^{2}} M \quad,\left|\varepsilon_{k}{ }^{T} \hat{h}_{i}^{*}\right|^{2}=\frac{p_{p}}{\left(1+p_{p}\right)^{2}} M
$$$$
\left|\hat{h}_{k}{ }^{T} \hat{h}_{k}^{*}\right|^{2}=\frac{p_{p}{ }^{2}}{\left(1+p_{p}\right)^{2}} M^{2} .
$$

\subsection{ZF precoding}

Form (9), we can derive the achievable sum rate lower bound of vector normalization with ZF precoding which is shown as follows:

$$
\begin{aligned}
R_{Z F} & =K \log _{2}\left(1+\frac{p_{f}\left|g_{k k}\right|^{2}}{1+p_{f} \sum_{i=1, i \neq k}^{K}\left|g_{k i}\right|^{2}+p_{f} \sum_{i=1}^{K}\left|\varphi_{k i}\right|^{2}}\right) \\
& \stackrel{(b)}{\approx} K \log _{2}\left(1+\frac{p_{f} \frac{M-K+1}{K} \frac{p_{p}}{1+p_{p}}}{1+\frac{p_{f}}{\left(1+p_{p}\right)}}\right) \\
= & K \log _{2}\left(1+\frac{M-K+1}{K} \frac{p_{f} p_{p}}{1+p_{p}+p_{f}}\right)
\end{aligned}
$$

Where $g_{k i}=\frac{\hat{h}_{k}{ }^{T} f_{i}}{\sqrt{K}\left\|f_{i}\right\|}, \varphi_{k i}=\frac{\varepsilon_{k}{ }^{T} f_{i}}{\sqrt{K}\left\|f_{i}\right\|},(b)$ apply the properties and the diversity order of ZF precoding ${ }^{[13]}$, we can obtain $\hat{h}_{k}{ }^{T} f_{i}=\left\{\begin{array}{ll}1 & i=k \\ 0 & i \neq k\end{array}{ }^{\prime}\left\|f_{k}\right\|^{2}=\frac{1+p_{p}}{(M-K+1) p_{p}}\right.$.

\section{SELECTION OF PRECODING SCHEME}

\subsection{Decision threshold}

Based on the above results from Form (10) and (11), we obtain it as follows:

$$
\begin{aligned}
\frac{\operatorname{SINR}_{Z F}}{\operatorname{SINR}_{\mathrm{MF}}}= & \frac{\frac{M-K+1}{K} \frac{p_{f} p_{p}}{p_{p}+1+p_{f}}}{\frac{M}{K} \frac{p_{f} p_{p}}{\left(p_{p}+1\right)\left(p_{f}+1\right)-\frac{1}{K} p_{f} p_{p}}} \\
& =\frac{M-K+1}{M}+\frac{K-1}{K} \frac{K}{M} \operatorname{SINR}_{Z F} \\
& =1+\frac{K-1}{M}\left(\operatorname{SINR}_{Z F}-1\right)
\end{aligned}
$$

Form (12), $\operatorname{SINR}_{Z F}<\operatorname{SINR}_{M F}$ when $\operatorname{SINR}_{Z F}<1$, i.e., $R_{Z F}<R_{M F} ; \operatorname{SINR}_{Z F}>\operatorname{SINR}_{M F}$ when $\operatorname{SINR}_{Z F}>1$, i.e., $R_{Z F}>R_{M F}$. Due to SINR is increasing function of the SNR in uplink, for (12) we expect that, in the high SNR region, ZF precoding is better than MF precoding; $\mathrm{MF}$ is better than $\mathrm{ZF}$ in the low SNR region. 
In order to facilitate the analysis of the experiment results, we assume that the length of uplink pilot sequences is equal to the number of users $\mathrm{K}$. When $S_{I N R}{ }_{Z F}=S I N R_{M F}$, it means that the performances of those two precoding scheme are comparable. Both ${ }^{R} Z F$ and ${ }^{R_{M F}}$ are concave function. Also, unlike ${ }^{R_{Z F}},{ }^{R_{M F}}$ is a monotonic increasing function. Thus, there exists two cross points: one is when the amount of users $K$ is one, and the other is given as follows:

$K_{\text {cross }}=\frac{(1+M) p_{f}}{p_{f}+1}-\frac{1}{p_{u}}$

When $\operatorname{SINR}_{Z F}=1$, we can obtain (13) as follows:

$\frac{M-K+1}{K} \frac{p_{f} p_{p}}{1+p_{p}+p_{f}}=1$

$K_{\text {cross }}$ can be regarded as a decision threshold of MF with ZF precoding. The performance of ZF precoding is better when the number of users $K$ less than ${ }^{K}$ cross and the performance of MF precoding is better when the number of users $\mathrm{K}$ is more than ${ }^{K}$ cross.

\subsection{Optimal number of users}

Let the number of uplink pilot symbols $\tau$ be equal to the number of users $K$, the spectral efficiency of two precoding are respectively denoted by $(1-\tau / T) R_{Z F}$ and $(1-\tau / T) R_{M F}$. The optimal number of spatial multiplexing users of MF and $\mathrm{ZF}$ are respectively denoted by $K_{Z F}{ }^{*}$ and $^{K_{M R T}}$. The purpose of this section is that the system can achieve the maximum transmission capacity when BS serves the number of users $K^{*}$.

In general, in the high SNR region, for example, when $S N R>>1$, we have $S I N R_{Z F}>S I N R_{M F}$. In this case, the optimal number of users for the Massive MIMO with $\mathrm{ZF}$ precoding to obtain maximum capacity is larger than MF precoding. The opposite is right in the low SNR region. As the number of BS antennas $M$ is raised, the forward SNR is decreased to zero, and then, we have $\operatorname{SINR}_{Z F} \approx \operatorname{SINR}_{M F}$. In this case, the optimal number of users for two precoding becomes comparable.

\section{SIMULATION RESULTS}

In this section, the effect of related parameters on the system performance with the algorithms is provided by numerical simulations. Channel is the modeled Rayleigh fading channel in the whole simulation process, and each element of the channel matrix $\mathrm{H}$ is i.i.d.CN $(0,1)$. For simplicity, we assume that the system is a single-cell scenario. Let the length of coherence interval be $\mathrm{T}=98$, then the length of uplink pilot symbols is $\tau=K$. The relevant parameters are shown in Table 1:

\begin{tabular}{ll} 
Table 1. Relevant parameters & \\
\hline Coherence time $T_{c}$ & $0.5 \mathrm{~ms}$ \\
Symbol interval $T_{s}$ & $71.4 \mathrm{us}$ \\
Useful symbol interval $T_{u}$ & $66.7 \mathrm{us}$ \\
Guard interval $T_{g}$ & $4.7 \mathrm{us}$ \\
Subcarrier interval $\Delta f$ & $15 \mathrm{KHz}$ \\
\hline
\end{tabular}

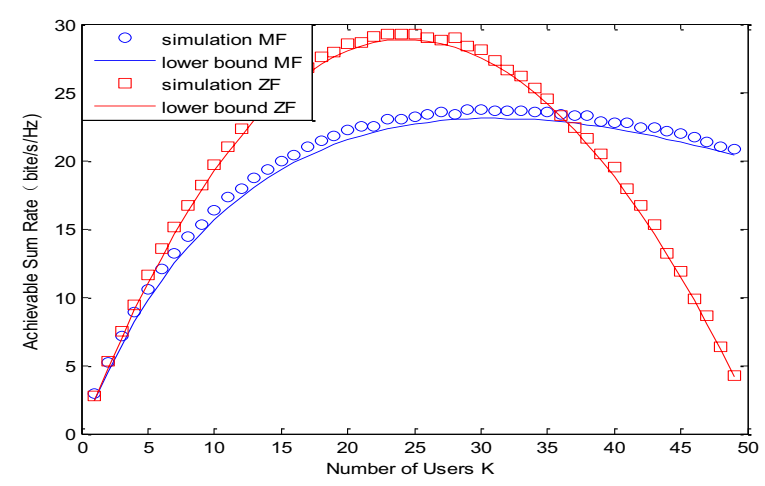

Figure 2. Performance comparison of $\mathrm{ZF}$ with $\mathrm{MF}$ precoding scheme, where $p_{f}=10 \mathrm{~dB}, p_{u}=-10 \mathrm{~dB}, \mathrm{M}=50$.

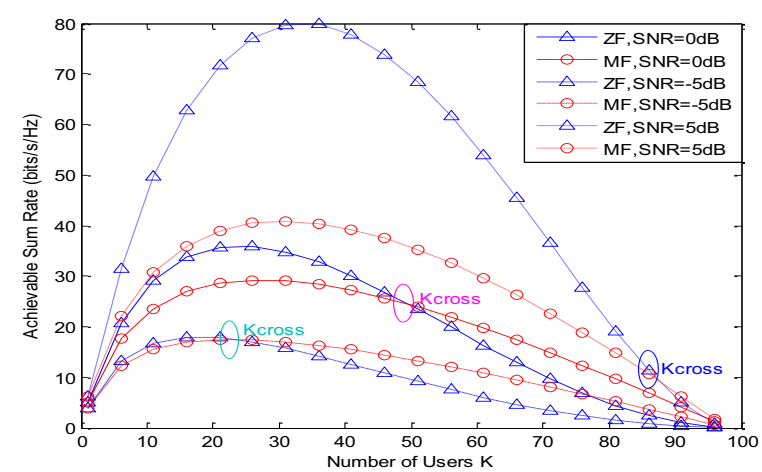

Figure 3. Achievable sum rate vs. number of users $\mathrm{K}$, where $p_{u}=0 \mathrm{~dB}$ and $\mathrm{M}=100$.

Figure 2 compares the performance between $\mathrm{ZF}$ and MRT precoding scheme by using the algorithm. From Figure 2, we can see that the ZF is better than MF precoding when the number of users is less; MF is better than ZF precoding when the number of users is more. Figure 3 compares the moving direction of cross point $K_{\text {cross }}$ (decision threshold) when SNR has different values by using the vector normalization for $\mathrm{ZF}$ and MF precoding. When $\mathrm{SNR}=-5 \mathrm{~dB}$ (in the low SNR region), $K_{\text {cross }}$ moved to the left; when $\mathrm{SNR}=5 \mathrm{~dB}$ (in the high SNR region), $K_{\text {cross }}$ moved to the right. From figure 3, it can be seen that we should 
choose the vector normalization MF precoding when the number of users $K$ is more than ${ }^{K}$ cross, and choose vector normalization $Z F$ precoding when the number of users $K$ is less than ${ }^{K}$ cross .

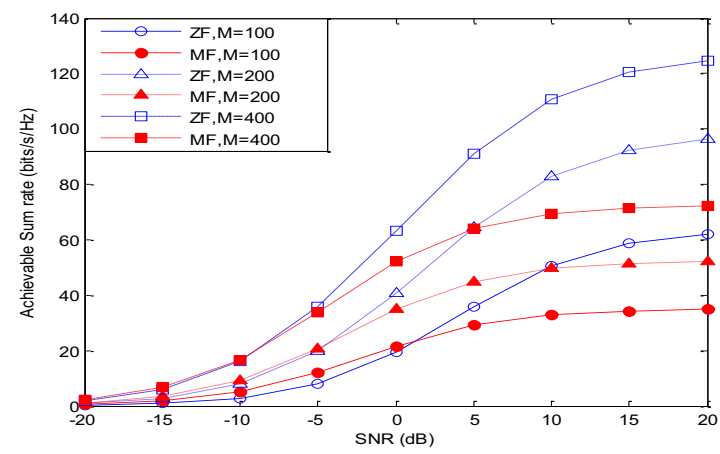

Figure 4. Achievable sum rate vs. SNR, where $\mathrm{K}=50$ and $p_{u}$ $=-10 \mathrm{~dB}$.

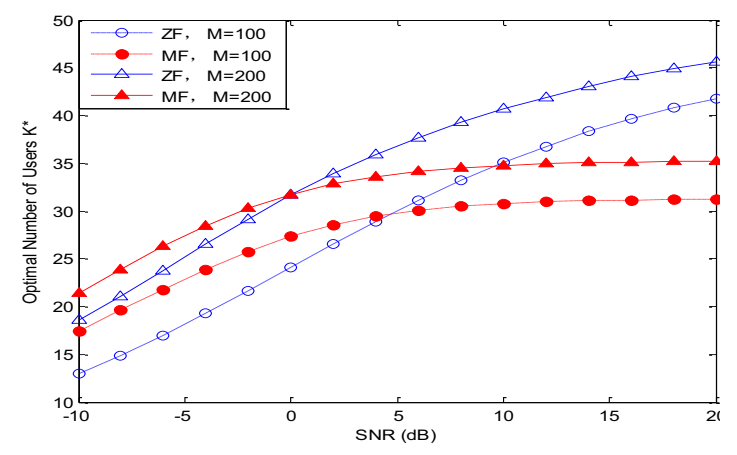

Figure 5. Optimal number of users $K^{*}$ vs. SNR, where $p_{u}$ $=0 \mathrm{~dB}$.

Figure 4 shows the achievable sum rate versus SNR. It is that $\mathrm{K}=50$ and $p_{u}=-10 \mathrm{~dB}$ when $M=100,200$ or 400 . We can see that the performance of system becomes better and better with the increasing $M$. In the low SNR region, MF precoding is better than ZF precoding with the vector normalization algorithm; in the high SNR region, ZF precoding is better than MF precoding. Figure 5 shows the optimal number of users $K^{*}$ versus SNR for massive MIMO system to achieve the maximal spectral efficiency. It is that $p_{u}=0 \mathrm{~dB}$ when $M=100$ or 200 . As we expected, in the low SNR region, the optimal number of users $K^{*}$ for massive MIMO with MF precoding is larger than that for the ZF precoding. The opposite is true in the high SNR region.

\section{CONCLUSIONS}

This paper offers the performance analysis and comparison of MF and ZF precoding scheme in a single-cell massive MIMO system for imperfect CSI, and derives the achievable sum rate of the two precoding schemes by suing the vector normalization algorithm. Theoretical analysis and numerical simulation have proved that the vector normalization with ZF precoding is better than MF precoding in the high SNR region, and the opposite is true in the low SNR region. Given the decision threshold for how to choose MF and ZF precoding, we can select the appropriate vector normalization algorithm according to the needs, and improve the system capacity of massive MIMO.

\section{ACKNOWLEDGMENT}

This paper is sponsored by the National Natural Science Foundation of China (GN: 61271276), National High Technology Research and Development Program of China (863 Program) (GN: 2014AA01A703, 2014AA01A705). The authors would like to thank anonymous referees for their very constructive comments and corrections of errors.

\section{REFERENCES}

[1] Larsson, E., Edfors, O., Tufvesson, F. \& Marzetta, T. 2014. Massive MIMO for next generation wireless system. IEEE Communication Magazine, 59(2): 186-195.

[2] Marzetta, T.L. 2010. Noncooperative cellular wireless with unlimited numbers of base station antennas. IEEE Transactions on Wireless Communications, 9(11) 3590-3600.

[3] Mehmood, Y., Afzal, W. \& Ahmad, F. 2013. Large scaled multi-user MIMO system so called massive MIMO systems for future wireless communication networks. IEEE Proceedings of the 19th International Conference on Automation and Computing (ICAC), pp: 83-86.

[4] Kobayash, M., Jindal, N. \& Caire, G. 2011. Training and feedback optimization for multiuser MIMO downlink. IEEE Transactions on Communications, 59(8): 2228-2240.

[5] Ngo, H.Q., Larsson, E.G. \& Marzetta, T.L. 2013 Energy and spectra efficiency of very large multiuser MIMO systerm. IEEE Transactions on Wireless Communications, 61(4): 1436-1449.

[6] Ngo, H.Q., Larsson, E.G. \& Marzetta, T.L. 2013. Massive MU-MIMO downlink TDD systems with linear precoding and downlink pilots. IEEE 51st Annual Allerton Conference on Communication, Control, and Computing, pp: 293-298.

[7] Yang, H. \& Marzetta, T.L. 2013. Performance of conjugate and zero-forcing beamforming in Large-scale. IEEE Journal on Selected Areas in Communications, 31(2): 172-179. 


\section{MATEC Web of Conferences}

[8] Chae, C.B., Kim, S.R. \& Heath, W. 2009. Network coordinated beam-forming for cell-boundary users: Linear and non-linear approaches. IEEE Signal Processing Society, 3(6): 1094-1105.

[9] Jose, J., Ashikhmin, A. \& Marzetta, T.L. 2011. Pilot contamination and precoding in multi-cell. IEEE Transaction on Wireless Communications, 10(8): 2640-2651.

[10]Huh, H., Caire, G. \& Papadopoulos, H.C. 2011. Achieving large spectral efficiency with TDD and not-so-many base-station antennas. IEEE Topical Conference on Antennas and Propagation in Wireless Communications, pp: 1346-1349.

[11]Lee, C.W., Chae, C.B., Kim, T. \& Choi, S. 2010 Network massive MIMO for cell-boundary users: from a precoding normalization perspective. IEEE Globecom Workshops, pp: 223-237.

[12] Tulino, A. M. \& Verdu, S. 2004. Random Matrix Theory and Wireless Communications. Now Publishers Inc., pp: 25-32.

[13] Wong, K. K. \& Pan, Z. 2008. Array gain and diversity order of multiuser MISO antenna systems. International Journal of Wireless Information Networks, 15(2): 82-89. 